\title{
Aprendizaje guiado por constreñimientos y modulación de la dificultad de práctica: Una propuesta de caso para el entrenamiento de la técnica en Gimnasia Rítmica
}

\author{
Constraints-led approach learning and practice complexity modulation: A case \\ proposal for technical training in Rhythmic Gymnastics \\ Carmen Ruano Masiá; Francisco.J Moreno \\ Universidad Miguel Hernández (España)
}

\begin{abstract}
Resumen. Uno de los puntos clave del entrenamiento deportivo es conocer cuándo un gesto técnico debe ser modificado, así como las estrategias que seguir para ello. Se ha comprobado cómo la interacción con diferentes materiales y espacios es adecuada para incentivar la búsqueda de soluciones motrices en los individuos, pero es importante conocer el estrés que supone para el sujeto la aplicación de cierta dificultad añadida. El objetivo del presente trabajo es mostrar la aplicación de la aproximación al aprendizaje guiado por constreñimientos para la modificación de cuatro gestos técnicos en Gimnasia Rítmica. La propuesta de aproximación consiste en un proceso por etapas a partir la detección de errores técnicos frecuentes. Se utiliza como ejemplo la aplicación en gimnasia rítmica, donde se pueden observar distintos tipos de errores susceptibles de cambio por diferentes motivos. Tras la propuesta práctica de una serie de ejercicios para tratar de corregir estos errores, se observa que un mismo ejercicio puede suponer diferentes niveles de carga en función del material y restricciones implementadas. La adecuación de la carga de práctica es importante para el diseño de tareas que tengan en cuenta las características del aprendiz, su nivel deportivo y los objetivos dentro del proceso de aprendizaje. Estos factores influirán en el nivel de carga que suponga la tarea para el deportista. Este tipo de trabajo es extrapolable a otros deportes, no obstante, es necesaria la realización de investigaciones prácticas en esta línea para recortar la distancia existente entre el conocimiento teórico y su aplicación práctica. Palabras clave: Entrenamiento, Técnica, Gimnasia Rítmica, Condicionantes, Variabilidad, Carga, Síndrome General de Adaptación, Aprendizaje.
\end{abstract}

\begin{abstract}
One of the key points in sports training is to know exactly when a technical movement needs to be modified, and the best strategies to do it. It has been shown that the interaction with different materials and spaces is an adequate strategy to enhance the search for motor solutions in athletes. However, it is also very important to know how an added difficulty affect to the performance of the individual. The main goal of this study is to provide an example of the application of the constraintsled approach in order to modify technical patterns of four technical gestures in Rhythmic Gymnastics. An example of the application in Rhythmic Gymnastics is presented. Some different kinds of errors to be modified because different reasons are shown. The proposal consists of some exercises designed in order to fix that mistakes. It can be seen that the same exercise may involve different practice loads depending on the features and the modulation of the task constraints, such as the material or the movement restrictions. Adaptation of the practice load is a main factor in the design of tasks when considering the characteristics of the learner, its sports level and the learning goals. We can apply this kind of work to other sports, but it is necessary to conduct practical investigations in this area to reduce the gap between the theoretical knowledge and its practical application.
\end{abstract}

Key words: Training, Technique, Rhythmic Gymnastics, Constraints, Variability, Load, Adaptation General Syndrome, Learning.

\section{Introducción}

La técnica deportiva es, junto con la táctica y la condición física, uno de los pilares fundamentales del rendimiento deportivo. En el proceso de entrenamiento de un deportista es muy importante el análisis y entrenamiento de la misma. Existe una controversia acerca de

Fecha recepción: 12-06-20. Fecha de aceptación: 07-07-21

Carmen Ruano Masiá

c.ruano@umh.es cuál debe ser y, si existe, el patrón técnico correcto en cada gesto deportivo (Glazier \& Mehdizadeh, 2019). Tradicionalmente se ha tratado de hacer coincidir los movimientos de los deportistas con los cánones de perfección técnica establecidos (Brisson \& Alain, 1996), pero cada vez está más asentada la idea de que cualquier gesto técnico que, dentro de unos límites reglamentarios, consiga los objetivos deseados y sea capaz de solucionar con éxito diferentes situaciones, es igualmente válido (Glazier \& Davids, 2009). Esta idea respalda las afirmaciones de Bernstein (1967) acerca de que la repetición idéntica de un movimiento en diferentes ocasiones es 
imposible, proponiendo que se pueden conseguir los mismos resultados por medio de distintas vías (repetición sin repetición). Por ello, mediante el trabajo de la técnica se debe dotar al deportista de oportunidades de exploración y búsqueda de soluciones ante las situaciones motrices (Button, Davids \& Schöllhorn, 2006).

Según Moreno \& Ordoño $(2009,2015)$ el aprendizaje motor en general, y el entrenamiento de la técnica en particular, se podría explicar a través de un proceso similar al descrito por el Síndrome General de Adaptación (SGA) propuesto por Selye (1956). Esta propuesta, encuadrada en los principios de la Teoría General de Sistemas Complejos (Von Bertalanffy, 1996), parte de la consideración del ser humano como un sistema complejo que tiende a buscar estados estables (de equilibrio) y que aprende mediante procesos de adaptación. De acuerdo con esta perspectiva, el comportamiento de los sistemas (los deportistas) a la hora de responder ante un cambio en el entorno (en este caso el esfuerzo o carga necesario para el aprendizaje o modificación de la técnica), seguiría el siguiente proceso: tras la aplicación de la carga se produciría un descenso en el rendimiento (fase de alarma), ya que el sujeto contaba con un patrón estable que se estaría comenzando a desestabilizar. Tras un período de aplicación del nuevo estímulo, el deportista comenzaría a adaptarse y a aumentar de nuevo el rendimiento hasta conseguir mejorar el rendimiento. $\mathrm{Si}$ las condiciones de entrenamiento se mantuvieran en el tiempo, el nuevo patrón pasaría a ser el patrón estable del individuo.

A través de interacciones con el material, el espacio, otros individuos y eventos durante el entrenamiento deportivo, los atletas pueden desarrollar la capacidad de explorar las posibilidades disponibles en un entorno perceptivo-motriz de posibles soluciones a una tarea (Davids, Araújo \& Brymer, 2016; Martín-Barrero y Camacho Lazarraga, 2020; Gil, Moreno, Claver, Moreno \& Del Villar, 2015). Este tipo de práctica ayudará al individuo a desarrollar técnicas individuales de búsqueda de soluciones en lugar de aprender una técnica determinada a la que todos los atletas deban asemejarse (Brisson \& Alain, 1996; Chow, Davids, Button \& Renshaw, 2016). Estas prácticas basadas en la exploración dentro de un rango de soluciones ayudarán al deportista a ser adaptativo, innovador y flexible. Los entrenadores son responsables de planificar y estructurar la práctica seleccionando de entre los condicionantes susceptibles de ser modificados aquellos que dirijan la atención del deportista a las fuentes de información relevantes (Renshaw, Davids, Newcombe \& Roberts,
2019). Para orientar a los entrenadores acerca de cuándo un gesto técnico debe ser modificado, Davids, Button \& Benett (2008) propusieron ciertos elementos clave:

I. Que el gesto no permita al deportista adaptarse a diferentes situaciones del deporte.

II. Que implique riesgo de lesión.

III. Que el gesto sea poco eficiente energéticamente hablando, o poco eficaz en el uso de la cadena cinética del movimiento.

IV. Que la ejecución repercuta de manera negativa en el resultado del deportista (por ejemplo, en la puntuación).

Además, las características tanto del deportista (edad, nivel deportivo, habilidades motrices, género, etc.) como de la tarea (nivel de dificultad, de habilidad del sujeto, etc.) intervienen en la atención del mismo respecto a la tarea, por lo que son un aspecto importante a tener en cuenta (Camacho Lazarraga, 2018). Un reto para los entrenadores es entender en qué punto del continuo de aprendizaje de una habilidad se sitúa el atleta en cada momento para ajustar la carga de trabajo de manera óptima y así maximizar los resultados (Renshaw, Davids, Newcombe \& Roberts, 2019).

Por último, cabe mencionar que es necesario aplicar los estímulos empleados con precaución, ya que una exposición continua y prolongada a los mismos puede llevar consigo diferentes riesgos, como alteraciones no deseadas en los patrones coordinativos del movimiento, posibles lesiones o comportamientos disruptivos (voluntad de cese de la práctica). Moreno \& Beneroso (2009) proponen unos principios generales para el diseño de tareas de modificación de la técnica basadas en esta perspectiva:

I. Determinar gesto técnico y condicionantes de ejecución.

II. Analizar las dinámicas intrínsecas del deportista.

III. Establecer el aspecto a trabajar, la metodología y el nivel de carga: diseño de ejercicios.

IV. Evaluar los resultados de la intervención que se está llevando a cabo.

En el presente artículo se presenta una ejemplificación de este tipo de trabajo basado en las propuestas y los criterios comentados anteriormente. Se parte de la detección de un error técnico susceptible de cambio y la aplicación de una carga de práctica para la modificación del mismo basado en los principios comentados. La propuesta se enmarca en el deporte de la Gimnasia Rítmica. La Gimnasia Rítmica es un deporte olímpico femenino en el que se realizan ejercicios individuales o en conjunto con el manejo de aparatos (cuer- 
da, pelota, aro, mazas y cinta) y con soporte musical. Se trata de una modalidad multifactorial, en cuyo rendimiento toman partido numerosos factores como la flexibilidad, la fuerza, la capacidad de salto, las características antropométricas o la coordinación (Coppola,Albano, Sivoccia \& Vastola, 2020; Ruano \& Cejuela, 2020). Se trata de un deporte en el que la técnica es uno de los principales factores de rendimiento (Cavallerio, Wadey \& Wagstaff, 2016; Douda, Toubekis, Avloniti \& Tokmakidis, 2008), por lo que la corrección en la ejecución de la misma será uno de los principales objetivos del entrenamiento. De acuerdo con el código de puntuación, existen diferentes grupos de elementos que las gimnastas deben ejecutar en un ejercicio de la modalidad individual: Dificultades corporales, de aparato, pasos de danza y elementos dinámicos con rotación (FIG, 2017). En el presente trabajo principalmente nos vamos a centrar en las dificultades corporales, que a su vez se encuentran divididas en equilibrios, giros y saltos. El análisis cinemático de la técnica en este deporte es uno de los principales campos de estudio en el que están surgiendo nuevas investigaciones al respecto, en especial en los elementos de salto (Coppola et al., 2020), encontrando menos referencias acerca del estudio de los equilibrios y los giros.

En los últimos años, se han establecido diferentes pautas en el diseño de ejercicios para la adquisición y el entrenamiento de habilidades deportivas atendiendo a distintas metodologías (ver Reinshaw et al. 2019). No obstante, son pocas las aproximaciones que hayan incluido en los criterios propuestos para el diseño de ejercicios, pautas o criterios que permitan establecer el nivel de carga de práctica que una determinada tarea supone para el aprendiz (Moreno \& Beneroso, 2009). El principal objetivo de este trabajo es elevar una propuesta de aplicación del entrenamiento guiado por constreñimientos y basada en los criterios de control de la carga de práctica en el deporte de la gimnasia, incluyendo la ejemplificación de ejercicios concretos. Partiendo de casos particulares de la ejecución técnica de ciertos elementos corporales, se propondrá ejercicios basados en el aprendizaje por constreñimientos para la corrección de errores técnicos proponiendo distintos niveles de carga de práctica. Se espera que esta propuesta pueda servir para estimular la aplicación de los criterios establecidos en la elaboración de otras propuestas en otras situaciones de este deporte o a otras modalidades deportivas. Cabe remarcar que, en este caso, se ha optado por ejemplos que no estarían orientados a la iniciación deportiva, en la que predominaría el trabajo de coordinación y el juego motriz, sino que se trata de una propuesta más enfocada a la práctica deportiva en niveles competitivos donde se busca un rendimiento más elevado y una mayor especificidad de los gestos técnicos.

\section{Características del caso y procedimiento de consenso con expertos}

Para ilustrar la propuesta de intervención, se ejemplifica con la participación de una gimnasta de 13 años (estatura: 1,44 m; masa corporal: $32 \mathrm{~kg}$ ), de nivel nacional absoluto. Esta gimnasta contaba con 7 años de experiencia deportiva, 6 de ellos en competición nacional.

Para la detección de errores y selección de las habilidades técnicas en las que centrar el trabajo, se contó con el asesoramiento de un equipo técnico responsable del entrenamiento de la participante, compuesto por dos entrenadoras de nivel internacional, así como por una preparadora física especializada en Gimnasia Rítmica. Tras las reuniones previas para la preparación de los ejercicios, se consensuó la aplicación de los siguientes criterios para la selección de habilidades:

I. Habilidades en las que existiera un error técnico observable, que además fuera común en otras gimnastas.

II. Habilidades de equilibrio, giro o técnica básica de aparato. Se eliminaron las dificultades de salto ya que los principales errores en ellos se derivan de una falta de fuerza explosiva y de flexibilidad (Di Cagno et al., 2008), errores cuya solución pasa por la vía de la preparación física. También se eliminaron los gestos de aparato más específicos (ya que estos variarán mucho de una a otra gimnasta y de uno a otro aparato, lo que dificultaría la generalización de la propuesta).

III. Habilidades cuya correcta ejecución pudiera servir como punto de partida para conseguir aprender elementos técnicos de mayor valor.

La aplicación de estos criterios se concretó en la selección de una serie de elementos que recogieran las características comentadas para para incluirlos en la propuesta. Es necesario señalar que estos elementos son seleccionados a modo de ejemplo entre otras posibilidades o elementos que también podrían reunir dichos criterios. Así, a efectos del presente trabajo se han seleccionado los siguientes elementos:

I. Equilibrio tono lateral

II. Equilibrio penché

III. Giro penché

IV. Técnica básica de lanzamiento 


\section{Descripción de la propuesta práctica}

A continuación, y en base a las características definidas en el apartado anterior, se presenta la propuesta de ejercicios para la modificación de errores en las situaciones seleccionadas.

Todos los ejercicios planteados fueron consensuados y elaborados en base a las recomendaciones del comité de expertas indicado en el apartado anterior. Todos los ejercicios de la propuesta fueron probados previamente por con gimnastas de competición para así asegurar la viabilidad de realización de los mismos. Además, a partir de la propuesta de aprendizaje guiado por constreñimientos se contó con este comité para determinar la adecuación del nivel de carga y la progresión de los ejercicios. En la tabla 1 se presenta un resumen de los ejercicios. miento en gimnasia rítmica (Donti, Bogdanis, Kritikou, Donti, \& Theodorakou, 2016).

\section{Condicionantes de la ejecución}

La ejecución de este elemento técnico está condicionada por la amplitud de movimiento de las caderas, ya que el ángulo de apertura de piernas debe ser próximo a $180^{\circ}$ en el plano frontal. Según Vernetta, PeláezBarrios \& López-Bedoya (2020), la flexibilidad, en especial de hombros y caderas, es determinante en el rendimiento en esta modalidad deportiva. Por otra parte, también destacamos la fuerza isométrica necesaria para mantener la pierna elevada en dicha posición durante el segundo que debe mantenerse fijada (fuerza de musculatura del tren inferior: cuádriceps, abductores, psoas ilíaco y fuerza de la musculatura abdominal y lumbar)

Para este elemento, el/la ejecutante debe tener una buena técnica de equilibrio, basada en el mantenimiento de una posición estática sobre los metatarsos. Esta técnica es la base para la mayoría de elementos técnicos en Gimnasia Rítmica, y su éxito dependerá en gran medida de una correcta colocación y distribución del peso corporal, alineado y con el centro de gravedad proyectado sobre la base de sustentación del equilibrio, que será la planta del pie al completo o el tercer metatarso, en función de si el equilibrio se realiza sobre la planta o sobre la media punta (Douda

\section{Ejemplo 1: Equilibrio Tono Lateral}

Se ha seleccionado el gesto técnico corporal «Equilibrio spagat lateral sin ayuda de la mano», también conocido como «tono lateral», cuya ejecución se puede observar en la figura 1.

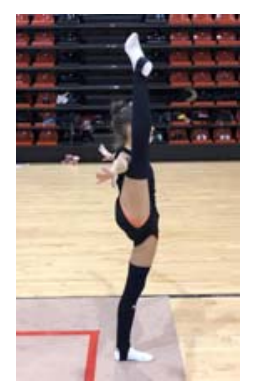

Figura 1. Equilibrio spagat lateral sin ayuda de la mano o equilibrio tono lateral

Según Cheng, Chang, Ju \& Tsao (2017), el equilibrio es uno de los principales factores de rendimiento en Gimnasia Rítmica. Además, la elevación lateral de la pierna es uno de los principales predictores del rendi- et al. 2008, Watson et al., 2017). La gran cantidad de movimientos que implican cambios de eje, de posición o de rotación que se dan en este deporte, dificultan la capacidad de mantenimiento del equilibrio. Las gimnastas deben ser capaces de integrar y coordinar la información recibida a través de las vías vestibular, visual y somatosensorial para orientarse en el espacio y llevar a cabo las tareas requeridas con éxito (Yeh, Chang \& Chen, 2019).

Según el código de puntuación (Féderation Internationale de Gimnastique (FIG), 2017), la habilidad debe mantenerse al menos un segundo con un ángulo de apertura próximo a $180^{\circ}$, y debe seguir los siguientes criterios:

o Apoyo sobre los metatarsos del pie.

o No puede haber movimientos del pie de base durante el equilibrio o al finalizar el mismo.

o La pierna debe encontrarse alineada desde la cadera con el tronco de la gimnasta. 


\section{Errores frecuentes}

Uno de los errores más frecuentes que se suelen encontrar en la ejecución de esta dificultad de equilibrio es la colocación incorrecta de la cadera, observando una anteversión que lleva consigo una desalineación de la pierna elevada con el tronco. Este error, además de resultar en una reducción de puntuación técnica, puede conllevar un riesgo de sobrecarga muscular de la espalda, lo cual sería negativo debido a la elevada tensión ya producida en las gimnastas por la realización de una gran cantidad de elementos técnicos que la provocan (Agopyan, 2021).

En la Tabla 2 se puede observar el resumen de las características de la ejecución y la propuesta de intervención con referencia a diferentes niveles de carga en la aplicación del ejercicio.

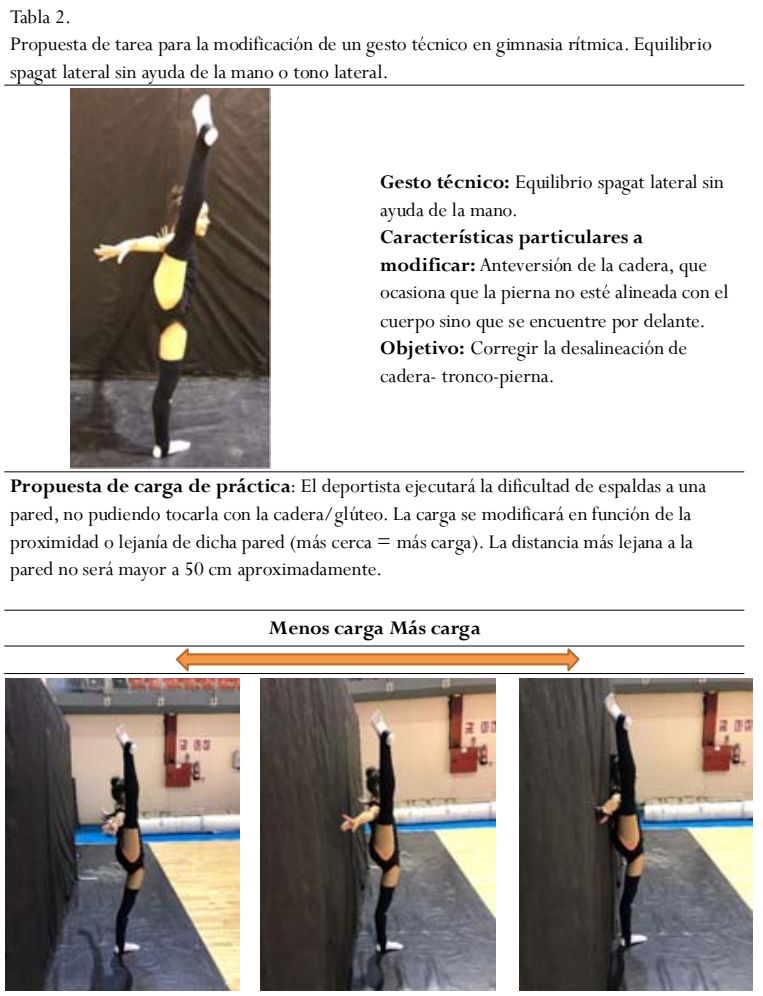

\section{Ejemplo 2: Equilibrio Penché}

Se ha seleccionado el gesto técnico corporal «equilibrio en penché», cuya ejecución se puede observar en la imagen de la figura 2.

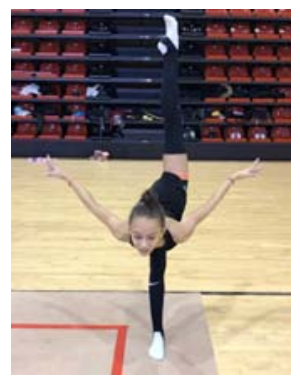

ondicionantes de la ejecución

La ejecución de este elemento técnico está condicionada en gran medida por la flexibilidad de caderas (a nivel anteroposterior), ya que la elevación de la pierna que se debe realizar debe formar como mínimo un ángulo de apertura de piernas de $180^{\circ}$ en el plano sagital. La flexibilidad dorsal, un factor relevante en esta modalidad deportiva (Agopyan, 2021) también es un punto a tener en cuenta, ya que la posición del tronco debe colocarse entre la pierna de base y la horizontal. Por otra parte, también destacamos la fuerza necesaria para mantener la pierna elevada y fijada en dicha posición.

Según el código de puntuación (FIG, 2017), la habilidad debe mantenerse claramente fijada, con un ángulo de apertura mínimo de $180^{\circ}$, y sin movimientos del pie de base durante el equilibrio o al finalizar el mismo. A pesar de que no lo indique de manera expresa el Código de Puntuación, es recomendable que la gimnasta finalice la ejecución con pies juntos y que, durante la misma, ambos brazos formen una línea paralela al suelo.

\section{Errores frecuentes}

Uno de los errores más frecuentes que encontramos en la ejecución de esta figura es la desalineación de los hombros, no formando una línea paralela al suelo. Este error suele ocasionarse debido a una activación muscular dorsal insuficiente en la parte opuesta de la espalda a la pierna que se eleva. Este error, además de resultar en una reducción de la puntuación técnica, dificultará el mantenimiento del equilibrio y puede conlle-

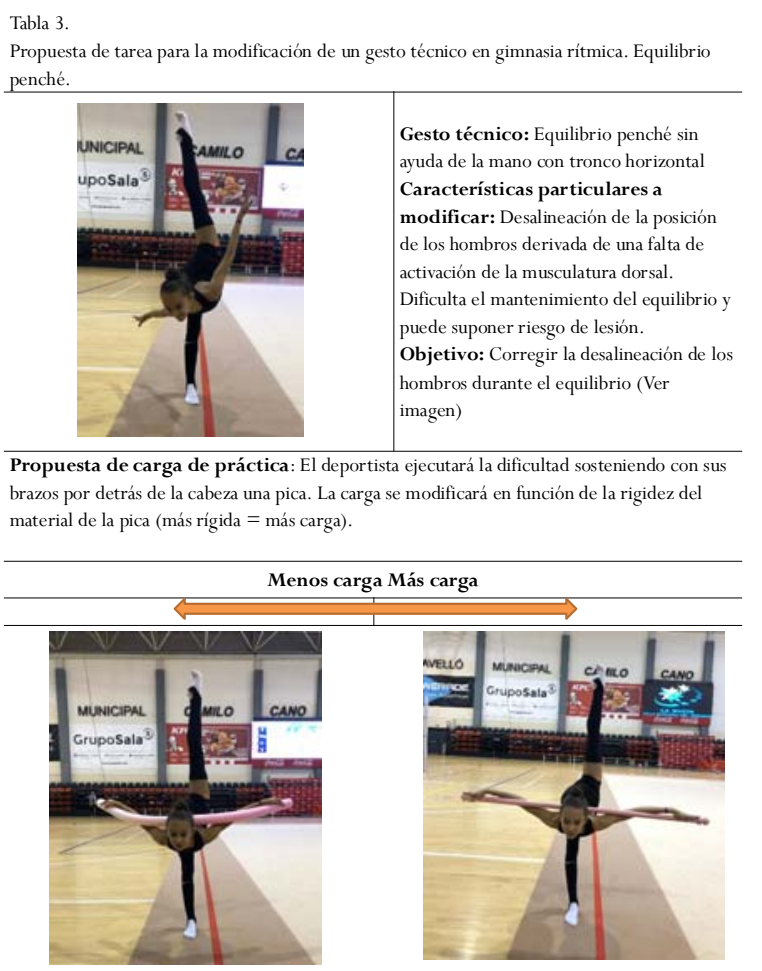


var un riesgo de sobrecarga y lesión muscular en la espalda debido a la descompensación de la fuerza que ejercen ambos lados de la misma, lo que puede llevar a incrementar el riesgo de incidencia de lesión en la espalda, que ya de por sí es elevado en esta modalidad debido a la hiperextensión de espalda que requieren la mayor parte de elementos técnicos corporales (Agopyan, 2021).

En la Tabla 3 se puede observar el resumen de las características de la ejecución y la propuesta de intervención con referencia a diferentes niveles de carga en la aplicación del ejercicio.

\section{Ejemplo 3: Giro Penché}

El gesto técnico corporal «giro en penché sobre la punta del pie». Se basa en la realización de rotaciones en la posición que se observa en la figura 3.

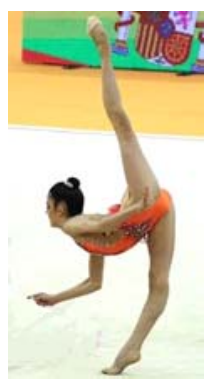

Figura 3. Giro penché

Según Cheng et al. (2017), el equilibrio es uno de los principales factores de rendimiento en Gimnasia Rítmica. Se ha seleccionado este gesto ya que aúna las características técnicas de los equilibrios, pero añadiendo un paso más de complejidad.

\section{Condicionantes de la ejecución}

Para este elemento, además de una técnica de equilibrio depurada, será fundamental también la técnica de giro. En las dificultades de giro cobra más importancia si cabe la correcta alineación de todos los segmentos corporales, para que la proyección del centro de gravedad en el suelo se dé justo sobre los metatarsos del pie sobre el que se realiza el giro, el que actúa como base de sustentación, ya que esto es lo que hará que se mantenga el eje de rotación y no se provoque un vencimiento de la gimnasta hacia ninguno de los lados, produciéndose un desequilibrio. Por último, también es muy importante que exista un punto en el que la gimnasta fije su mirada durante la ejecución del giro. Según Chua, Sproule \& Timmons (2018), las bailarinas profesionales se caracterizan por mantener un foco de atención interno en la ejecución de los giros.

Según el código de puntuación (FIG, 2017), la gim- nasta debe completar al menos una rotación de $360^{\circ}$ con un ángulo de apertura de piernas igual o superior a $180^{\circ}$ en el plano sagital, y debe seguir los siguientes criterios:

o Forma fijada sin variaciones durante toda la rotación

o Ejecutar la dificultad sobre los metatarsos del pie de base, sin apoyo del talón en ningún momento hasta la finalización

o No puede haber desplazamiento durante la rotación o al finalizar la misma.

o Ambos brazos deben formar una línea paralela al suelo.

\section{Errores frecuentes}

Uno de los errores más frecuentes que se encuentran en la ejecución de esta dificultad corporal es la colocación errónea de la cadera (anteversión excesiva) que provoca que la pierna que se despega del suelo se oriente hacia un lateral y no hacia la vertical. Este error, además de resultar en una reducción de la puntuación técnica, dificultará el mantenimiento del equilibrio y puede conllevar un riesgo de sobrecarga y lesión muscular.

Otro error frecuente es el desplazamiento del centro de masas hacia atrás en la finalización de la rotación, provocando un pequeño desequilibrio y finalizando con el pie que se ha elevado por detrás del pie de base en lugar de a su lado. Este error resultará en una reducción de la puntuación técnica y dificultará el mantenimiento

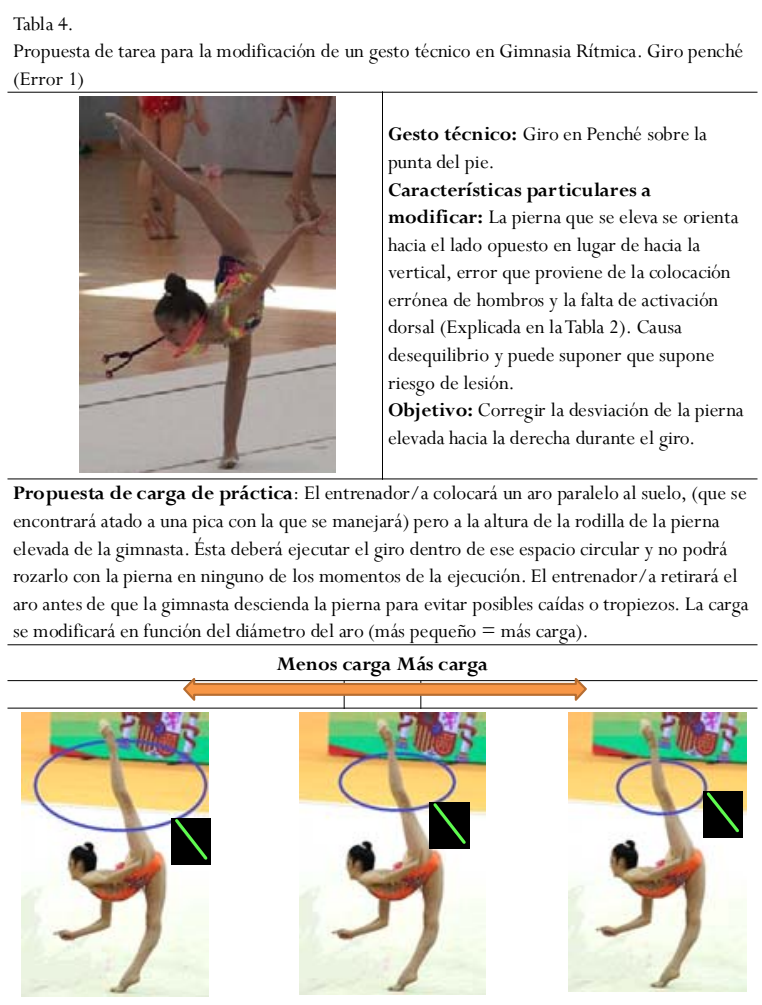


del equilibrio.

En las tablas 4 y 5 se puede observar el resumen de las características de la ejecución y la propuesta de intervención con referencia a diferentes niveles de carga en la aplicación de los ejercicios.

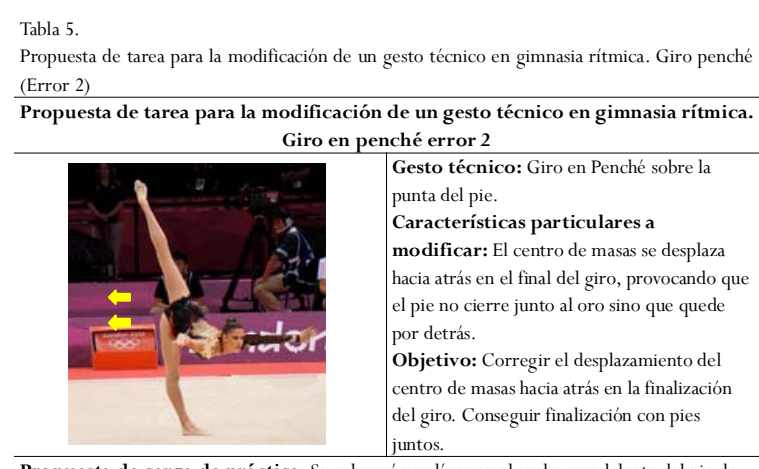

Propuesta de carga de práctica: Se colocará una línea en el suelo, por delante del pie de base. La gimnasta deberá ejecutar el giro y en la finalización tocar la línea con el pie que estaba elevado. La carga se modificará en función de la distancia entre la línea y el pie de apoyo (más \begin{tabular}{l} 
distancia $=$ más carga). \\
\hline
\end{tabular}

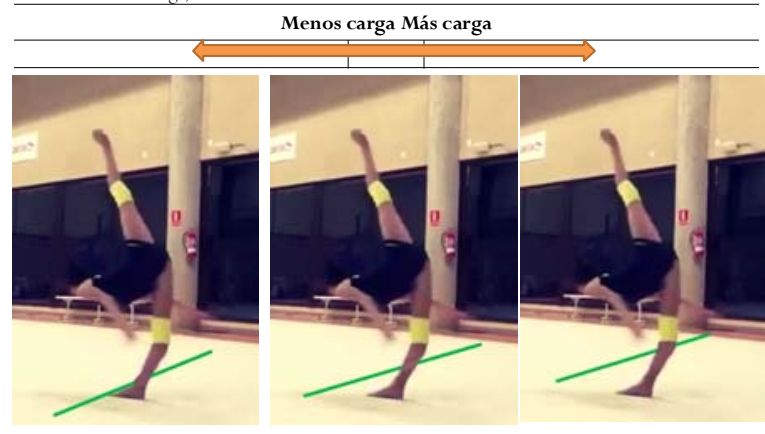

\section{Ejemplo 4: Lanzamiento de Aparato}

En este caso se ha seleccionado la técnica de lanzamiento de cualquier aparato, ya que es la base para la realización de todas las dificultades de riesgo obligatorias en los ejercicios, que consisten en la ejecución de diferentes rotaciones o elementos durante el tiempo de vuelo del aparato, y la posterior recepción del mismo con la mano o cualquier otra parte del cuerpo (en función del nivel técnico de la gimnasta).

\section{Condicionantes de la ejecución}

Para la ejecución de los lanzamientos, la gimnasta empleará su brazo dominante. Se trata de un movimiento de cadena cinética abierta en el que participa todo el cuerpo. Comienza con una flexión de rodillas y
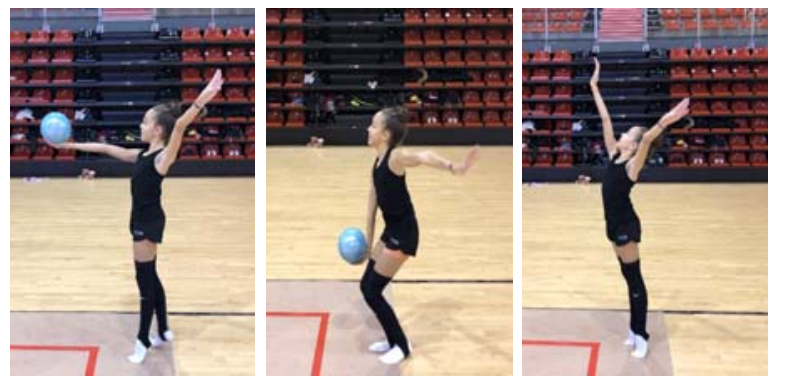

Figura 4. Serie de imágenes del proceso de un lanzamiento de pelota en gimnasia rítmica. un descenso del brazo que ejecutará al lanzamiento. A continuación, las rodillas se extenderán a la vez que se elevará el brazo sin flexionar el codo, para después lanzar el aparato. El proceso se puede observar en la serie de imágenes de la figura 4.

El Código de puntuación no hace referencia a deducciones en la puntuación derivadas de la ejecución correcta de la técnica de lanzamiento, más allá de la colocación errónea de los segmentos (deducción de 0.1 puntos). No obstante, una correcta ejecución técnica del movimiento permitirá el control de la trayectoria en vuelo: altura y distancia del lanzamiento, lo que definirá el éxito en la dificultad técnica a realizar. Los principales condicionantes que deben cumplirse son:

o Brazo del lanzamiento extendido en todo momento.

o Lanzamiento realizado a partir de la altura de la línea de hombros.

o Brazo no dominante acompaña el movimiento desde abajo hacia arriba o se mantiene en un lateral a la altura del hombro o ligeramente por encima.

o Ejecución del lanzamiento con los pies juntos (no siempre, pero es la base de la técnica)

o Finalización del movimiento con los pies juntos y sobre los metatarsos, sin apoyo del talón.

\section{Errores frecuentes}

Uno de los errores más frecuentes con los que nos encontramos es la flexión del codo a la hora de lanzar el aparato. Esto dificultará el control de la dirección del mismo, lo que reducirá las posibilidades de éxito en la ejecución de la dificultad que se esté realizando. Esto se traducirá en una trayectoria y altura incorrecta del lanzamiento, que puede afectar a su recepción o a los movimientos que se tuvieran que realizar durante el vuelo, ocasionando una posible recuperación incorrecta o incluso en una pérdida del implemento (con la consecuente deducción en la puntuación final que eso conlleva).

En la tabla 6 se puede observar el resumen de las características de la ejecución y la propuesta de intervención con referencia a diferentes niveles de carga en la aplicación de los ejercicios.

*En este caso se observa una progresión inversa en la rigidez de los materiales que en el Ejemplo 2. Esto se debe a una búsqueda del aumento de la sensibilidad propioceptiva. De esta manera, si se impide totalmente la flexión del codo, el/la deportista deberá realizar un esfuerzo menor para mantenerlo extendido, mientras que si el material empleado dificulta pero sí que la 


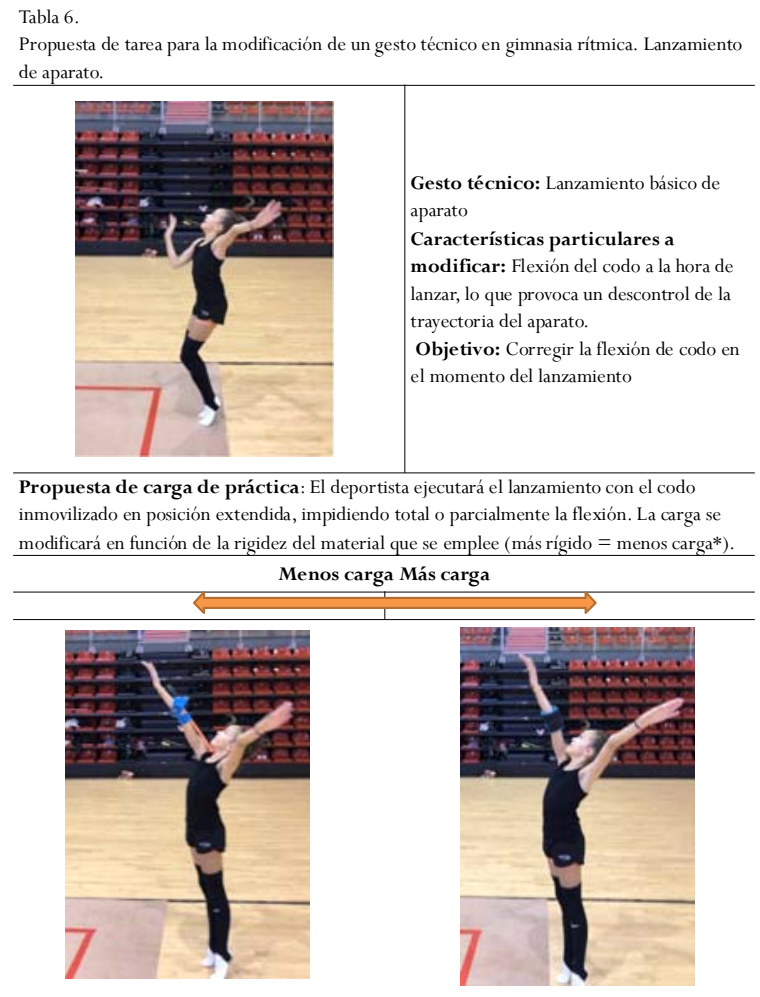

permite, la gimnasta deberá realizar un esfuerzo mayor por ejecutar el movimiento de manera correcta.

\section{Discusión y conclusión}

En el presente trabajo se presenta una propuesta de entrenamiento guiado por constreñimientos mediante los que se pueda aumentar en mayor o menor medida la dificultad de los ejercicios técnicos para modificar la conducta motora a partir de ejemplos de posibles errores. Se han empleado diferentes tipos de restricciones o constreñimientos, desde algunas más sencillas como el uso de una pared o una línea en el suelo hasta otras más complejas como el uso de picas, bandas elásticas o aros. No obstante, y por lo general, se propone la utilización de material deportivo básico de fácil acceso que se pueden implementar una gran cantidad de ejercicios y variantes útiles en el día a día. Encontramos escasos estudios similares como el de Moreno \& Beneroso (2008), en el que se realizan propuestas de entrenamiento técnico basadas en los mismos principios y que han resultado efectivas en otros deportes como, en ese caso, en el tenis.

En la propuesta, basada en los principios de la modificación de los constreñimientos, se han presentado modificaciones en los ejercicios para que éstos supongan niveles de carga diferentes en función del ejercicio en el que se apliquen. Previos autores han sugerido que el diseño de ejercicios debe permitir la emergencia de soluciones ajustadas al nivel del deportista (Chow et al, 2016). Vygotsky (1962) ya se refería a esta aproximación cuando conceptualizaba la Zona de Desarrollo Próximo, entendida como la distancia entre el nivel real del desarrollo, determinado por la capacidad de resolver independientemente un problema y el nivel de desarrollo potencial determinado a través de la solución de un problema. Así, por ejemplo, en uno de los casos se ha propuesto una progresión de materiales en función de su rigidez; una rigidez mayor suponía un nivel de carga más elevado mientras que en otro caso era a la inversa. Esto pone además el foco la importancia de tener en cuenta la interacción de las características del aprendiz con las características específicas de cada gesto técnico para saber cómo manejar la carga que suponen los constreñimientos introducidos para conseguir los resultados deseados, ya que en cada uno de ellos el modelo a seguir deberá de ser diferente. Por tanto, la adaptación de las tareas a las características del aprendiz, a su nivel de ejecución y al momento dentro del proceso de aprendizaje en el que se encuentre serán vitales en el éxito del proceso de entrenamiento, ya que en función de todos esos factores cada ejercicio supondrá una mayor o una menor carga (Renshaw et al., 2019).

A pesar de que el presente trabajo se basa en una propuesta de ejercicios en base a un modelo teórico de aprendizaje, se tomó como ejemplo un estudio de caso, en el que un grupo de expertas, en colaboración con los investigadores determinó una posible intervención sobre ciertos gestos particulares para una deportista en concreto. El uso de este tipo de ejercicios basados en una aproximación al aprendizaje guiado por constreñimientos se basa en principios que pueden ser comunes a otros deportes individuales en los que la técnica es uno de los principales factores del rendimiento, como pueden ser la Natación, el Patinaje Artístico, la Natación Sincronizada, elTenis, etc. Sería necesario analizar las características y condicionantes de la ejecución del ejercicio sobre el que se desea trabajar y seguir un procedimiento similar al mostrado en el presente trabajo (Renshaw et al., 2019). No obstante, según estos autores, la manipulación óptima de los condicionantes es un reto para los entrenadores, debido a que en muchas ocasiones es difícil traspasar las barreras entre la teoría y la práctica y conseguir una manipulación óptima de los mismos para obtener los resultados deseados.

Por otro lado, existe una gran cantidad de deportes en los que la técnica no es el fin en sí mismo (tal y como sucede en la mayoría de los deportes artísticos como la Gimnasia Rítmica), sino que es el medio que permite 
conseguir un fin, como por ejemplo encestar un tiro libre en baloncesto o marcar un penalti en fútbol. Este es el caso de la mayoría de los deportes colectivos, así como de otros individuales como los deportes cíclicos. Es en estos deportes donde se plasma de forma más evidente la controversia acerca de cuál debe ser y, si existe, el patrón técnico correcto en cada gesto deportivo. No obstante, Glazier \& Mehdizadeh (2019) comentan que esta situación puede ser común a diferentes niveles en otros deportes tradicionalmente considerados cerrados. Si bien es cierto que la presente propuesta se basa en ajustas los movimientos de las deportistas con los cánones técnicos, se debe tener en consideración que determinadas variaciones de los gestos técnicos, dentro de unos límites reglamentarios, pueden permitir alcanzar los objetivos deseados de forma válida (Glazier \& Davids, 2009). Así, aunque en otros deportes en los que el entrenamiento técnico en muchas ocasiones queda relegado a un segundo plano, el aprendizaje guiado por constreñimientos permite que el deportista explore y elabore el desarrollo de un patrón técnico ajustado a sus características intrínsecas, teniendo además aplicación no sólo al entrenamiento técnico sino a situaciones tácticas (Cantos \& Moreno, 2019). Por ejemplo, la capacidad de adaptación de la técnica de lanzamiento de un jugador de balonmano en función de las diferentes situaciones que se puedan dar en un partido (colocación de los adversarios, grado de fatiga, factores externos, etc.) será un aspecto determinante en su rendimiento, y el entrenamiento de esta capacidad de adaptación puede verse favorecido por el entrenamiento guiado por constreñimiento (FloresRodríguez \& Ramírez-Macías, 2020). Por un lado, en estos deportes también se debería analizar si la técnica ejecutada por los deportistas es la óptima para la consecución del fin principal (ya sea marcar un gol, optimizar la economía de carrera, etc.), o si por el contrario la modificación de ciertos patrones de movimiento podría incrementar el rendimiento en la acción (o evitar algún tipo de lesión). Por otro lado, la inclusión de condicionantes de la acción en el entrenamiento supone una metodología idónea para el trabajo del aspecto táctico, decisional e intencional, así como de asociación entre percepción y acción (Turvey, 1977).

Todo esto nos hace pensar que el trabajo basado en el aprendizaje guiado por constreñimientos puede ser un método de trabajo de la técnica en todo tipo de deportes, entre ellos la Gimnasia Rítmica. En el presente estudio se ha elaborado una propuesta que puede ser de utilidad para entrenadores a la hora de corregir algunos de los errores técnicos más frecuentes que observamos en este deporte. No obstante, es necesaria la realización de investigaciones prácticas en esta línea, para así recortar la distancia existente entre el conocimiento teórico y su aplicación al campo del entrenamiento.

Como línea de futuro derivada del presente trabajo, sería interesante introducir la propuesta realizada en un grupo de gimnastas de características similares a la deportista de estudio, aplicándola durante un período de tiempo para poder comparar la ejecución previa y posterior a la intervención y así poder contrastar la efectividad de la propuesta de una manera más robusta.

\section{Referencias}

Agopyan,A. (2021)An analysis of movements with or without back bend of the trunk or large hip extension in 1st Juniors' Rhythmic Gymnastics World Championship2019. Is there injury risk for gymnasts?, International Journal of Performance Analysis in Sport, 21:1, 108-125, DOI: 10.1080/24748668.2020.1850038

Bernstein,A.(1967). The coordination and regulation of movements. London: Pergamon Press.

Brisson, T.A., \& Alain, C. (1996). Should common optimal movement patterns be identified as the criterion to be achieved? Journal of Motor Behavior, 28(3), 211-223.

Button, C., Davids, K., \& Schöllhorn,W. (2006). Coordination profiling of movement systems. In: Davids, K., Bennett, S., \& Newell, K. (eds), Movement system variability, Champaign, IL: Human Kinetics, pp. 133-152.

Cantos, J., \& Moreno, F. (2018). Pedagogía no lineal como método de enseñanza de los comportamientos tácticos en los deportes de equipo, aplicación al rugby (Nonlinear pedagogy as a method of teaching tactical behaviors in team sports, rugby application). Retos, 35, 402-406. https: / / doi.org/10.47197/retos.v0i35.63508

Camacho Lazarraga, P. (2018). Efecto del foco atencional sobre el aprendizaje de las habilidades deportivas individuales (Effect of attentional focus on the learning of individual sports skills). Retos, 36(36), 561-566. https:// doi.org/10.47197/retos.v36i36.64428

Cavallerio, F., Wadey, R., \& Wagstaff, C. R. D. (2016). Understanding overuse injuries in rhythmic gymnastics: a 12-month ethnographic study. Psychology of Sport and Exercise, 25, 100-109. https://doi.org/10.1016/ j.psychsport.2016.05.002

Chen, H. Y., Chang, H. Y., Ju, Y. Y., \& Tsao, H. T. (2017). Superior short-term learning effect of visual and sensory organisation ability when sensory information is unreliable in adolescent rhythmic gymnasts. Journal of 
Sports Sciences, 35(12), 1197-1203.

Chow, J.-Y., Davids, K., Button, C., \& Renshaw, I. (2016). Nonlinear pedagogy in skill acquisition: An introduction. London: Routledge.

Chua, T. X., Sproule, J., \& Timmons, W. (2018). Effect of Skilled Dancers' Focus of Attention on Pirouette Performance. Journal of Dance Medicine \& Science, 22(3), 148 159. doi:10.12678/1089-313x.22.3.148

Coppola, S., Albano, D., Sivoccia, I., \& Vastola, R. (2020). Biomechanical analysis of a rhythmic gymnastics jump performed using two run-up techniques. Journal of Physical Education \& Sport, 20(1).

Davids, K., Araújo, D., \& Brymer, E. (2016). Designing affordances for health-enhancing physical activity and exercise in sedentary individuals. Sports Medicine, 46(7), 933-938.

Davids, K., Button, C. \& Bennett, S. (2008). Dynamics of Skill Acquisition: A Constraints-led Approach. Champaign, Illinois. Human Kinetics.

Donti, O., Bogdanis, G. C., Kritikou, M., Donti, A., \& Theodorakou, K. (2016). The relative contribution of physical fitness to the technical execution score in youth rhythmic gymnastics. Journal of Human Kinetics, 51(1), 143-152. https: / /doi.org/10.1515/hukin-2015-0183

Douda H., Toubekis A., Avloniti A. \& Tokmakidis S. (2008). Physiological and anthropometric determinants of rhythmic gymnastics performance. International Journal of Sports Physiology Performance. 2008;3(1):41-54.

Féderation Internationale de Gimnastique. (2017). Código dePuntuación Gimnasia Rítmica 2017-2020. Disponible en: http: / / aragongym.com/2016/NORMATIVA / RG_CoP\%202017-2020_sp.pdf

Flores-Rodríguez, J., \& Ramírez-Macías, G. (2020). Pedagogía no lineal en balonmano. Defensa zonal de una y dos líneas en partidos reducidos. Retos, 39, 604-613. https://doi.org/10.47197/retos.v0i39.79640

Gil, A., Moreno, M. P., Claver, F., Moreno, A., \& DelVillar, F. (2015). Manipulación de los condicionantes de la tarea en Educación Física: Una propuesta desde la pedagogía no lineal. Retos, 29, 22-27. https: / /doi.org/10.47197/ retos.v0i29.34612

Glazier, P. S., \& Davids, K. (2009). Constraints on the complete optimization of human motion. Sports medicine (Auckland, N.Z.), 39(1), 15-28. https://doi.org/ 10.2165/00007256-200939010-00002

Glazier, P.S. \& Mehdizadeh, S. (2019). In search of sports biomechanics' holy grail: Can athlete-specific optimum sports techniques be identified? Journal of Biomechanics, 94, 1-4

Martín-Barrero, A., \& Camacho Lazarraga, P. (2020). El diseño de tareas de entrenamiento en el fútbol desde el enfoque de la pedagogía no lineal (Design of training tasks in football from the nonlinear-pedagogy approach). Retos, 38(38), 768-772. https://doi.org/10.47197/ retos.v38i38.76612

Moreno, F.J., \& Ordoño, E.M. (2015).Variability and practice load in motor learning. RICYDE. Revista internacional de ciencias del deporte, 39(11), 62-78. http://dx.doi.org/ 10.5232/ricyde2015.03905

Moreno, F. J. \& Ordoño, E. M. (2009). Aprendizaje motor y síndrome general de adaptación. Motricidad. European Journal of Human Movement, 22, 1-21

Moreno, F.J \& Beneroso, F. (2009). Criterios metodológicos en el trabajo de la técnica basados en el Síndrome General de Adaptación. E-coach, Real Federación Española deTenis [Consultado el 5 de abril 2019].

Renshaw, I., Davids, K., Newcombe \& D., Roberts, W. (2019). The Constraints-Led Approach. Principles for Sports Coaching and Practice Design. Routledge.

Ruano-Masiá, C., \& Cejuela-Anta, R. (2020). Evaluación de los principales factores de rendimiento en gimnasia rítmica. Comparación entre diferentes niveles. Cultura, Ciencia y Deporte, 15(44), 65-175.

Selye, H. (1956). The stress of life. New York. McGraw-Hill. Turvey, M. T. (1977). Preliminaries to a theory of action with reference to vision. Perceiving, Acting and Knowing, 211-265.

Vernetta, M., Peláez-Barrios, E.M., \& López-Bedoya, J. (2020). Systematic review of flexibility tests in gymnastics. Journal of Human Sport and Exercise, in press. doi:https://doi.org/10.14198/jhse.2022.171.07

Von Bertalanffy, L. (1996). Teoría general de los sistemas.

Wallace, S. (1997). Dynamic Pattern Perspective of Rhythmic Movement:A Tutorial. en Zelaznik, H.N. (Ed.) Advances in Motor Learning and Control. Illinois. Human Kinetics Watson, T., Graning, J., McPherson, S., Carter, E., Edwards, J., Melcher, I., \& Burgess, T. (2017). Dance, balance and core muscle performance measures are improved following a 9-week core stabilization training program among competitive collegiate dancers. International journal of sports physical therapy, 12(1), 25.

Yeh,T.T., Chang, H.Y., Ju,Y.Y., \& Chen, H.Y. (2019). The use of a dual-task paradigm to identify superior sensory organisation ability in rhythmic gymnasts. Journal of sports sciences, 37(7), 772-778. Doi: 10.1080/ 02640414.2018.1526759 